sively over several generations. Progressive selection yields recombinant strains with multiple gene changes so it is virtually impossible to determine the effect of any one particular mutation on the nervous system.

In Proc. US Nat. Acad. Sci. $(58,1112 ; 1967)$, Benzer now reports the isolation in one generation of two mutants with changed phototactic behaviour. $\mathrm{He}$ devised a most ingenious selection technique, analogous to the counter current procedure used to separato mixtures of molecules, to isolate the two mutants. In essence, this involves putting Drosophila at the bottom of a tube over which is inverted a second tube. After arousing the flies a light is shone at either end of the joined tubes and the flies move in response to it. After 1 min the tubes are separated and new tubes attached to each and the process repeated for fifteen cycles. In model experiments, mixtures of wild type and wing deficient mutant flies, which also have poor phototactic response, were separated in this way.

Benzer then mutagenized with ethyl methane sulphonate (EMS) a population of male flies-strain Canton, Ohio-Standard - which he found forms a homogeneous population with strong phototactic response and then mated them with virgin $X$ chromosome attached females. He used this unusual female strain as a genetic trick to ensure that all the male progeny received their $X$ chromosome from the mutagenized male parent, not, as is usual, from the female. The twenty-six male progeny of this mating which lacked phototactic response were isolated by the counter current procedure. After a second mating of these males individually with virgin $X$ attached females to distinguish between true behavioural mutants and flies which, although phenotypically non-phototactic, are genetically normal, he was left with two sex-linked mutants which had lost phototactic behaviour in one generation. Furthermore, the site of these two mutations must differ, for one mutant has abnormal wing veins whereas the other appears normal.

Phototaxis is obviously a complex behavioural pattern involving reception of stimuli, transmission and integration of nervous signals and the generation of a motor signal telling the fly to move towards light. A mutation altering any of these elements would alter phototaxis, but by isolating many different mutants it should be possible to analyse the wiring diagram of the nerve circuits and the biochemistry of the impulse transmissions involved in phototaxis. Moreover, the counter current selection method can obviously be adapted for such stimuli as gravity, odour and sound and, by using a two stage procedure, any two factors can be selected for independently. Although this remarkable work is only a beginning, it provides a method for the isolation of many behavioural mutants and ultimately the genetic analysis of behaviour.

\section{Family of Proteins}

\section{from our Molecular Biology Correspondent}

Certain families of proteins-particularly the haemoglobins, the cytochrome cs and the lysozymes-have been the target of biochemical attacks in depth on broad fronts. Large numbers of these proteins have been sequenced, and it has been possible to draw broad conclusions about the number of positions in the chain which must remain invariant and the nature of the substitutions permissible elsewhere, and here and there some information has accrued on the effects of particular substitutions on aspects of the activity and structure.

Sequence data on a considerable number of lysozymes are available, thanks mainly to Jollès and his collaborators, and his latest review (Bull. Soc. Chim. Biol., 49, 1001 ; 1967) draws together the large number of facts which have been uncovered. The vertebrate lysozymes are basic proteins, which are substantially similar in amino-acid composition, but show considerable differences in their sequences. It is possible to make some assertions at least about residues not specifically involved in the activity or in maintaining the conformation. Thus, one lysozyme (duck egg) has been described which is devoid of histidine. This appears to put paid to schemes involving histidine in the enzymic mechanism. The residues near the $\mathrm{N}$-terminal end of the chain are also not involved, since two or three of these can be removed with an aminopeptidase without detriment to the function. The X-ray structure of hen's egg lysozyme indicates that three of the six tryptophan residues are clustered around the active centre; Jollès reports that two tryptophans, including one of the above three, try-108, can be chemically destroyed without observable diminution of activity. Even the number of disulphide bonds is variable. In hen's egg lysozyme, which by virtue of the X-ray structure is implicitly taken as the norm, there are four, in human lysozymes (milk and tears) three and in goose egg lysozyme two. This is also the order of thermal stability of the enzymes, the last, which also has a low tryptophan content, being very unstable. The structural lability is correlated with high enzymic activity, which one would like to believe reflects a readily responsive conformation (in terms, for example, of the induced-fit concept). A bacteriophage lysozyme, sequenced last year by Inouye and Tsugita, has the same substrate specificity as the mammalian lysozymes, but has very different sequence and composition, and may well be unrelated.

The most unexpected observation comes now from Brew, Vanaman and Hill (J. Biol. Chem., 242, 3747; 1967), who have determined the sequence of the milk protein $\alpha$-lactalbumin. This is a component of the lactose synthetase system, and catalyses the formation of the same glucopyranosyl linkage which is broken by the lysozymes. It is astonishing to find an extensive and unmistakable sequence homology with hen's egg lysozyme. Forty of the 123 residues of $\alpha$-lactalbumin are identical with those in the corresponding positions in lysozyme. A further 27 residues are of similar type ("conservative" substitutions). In both proteins there are four disulphide links; two of these only have so far been determined in lactalbumin, and these are identical to their counterparts in lysozyme. 'To achieve the best homology, two double-residue and four single-residue gaps must be allowed in the lactalbumin sequence, and two double-residue gaps in lysozyme. A remarkable point of difference is that, despite the homologies, $\alpha$-lactalbumin is an acidic protein, whereas lysozyme is basic (the isoelectric points are $p \mathrm{H} 5$ and 10.5). It seems then that the two proteins derive from a common precursor, and that a duplication of genes occurred at some stage, which was followed by the separate evolution of $\alpha$-lactalbumin in the mammals. 